\title{
Psychometric Properties of a Modified Balance Evaluation Systems Test
}

\author{
(1) Pedram Pourmahmoudian1, (1) Ali Asghar Noraste1, (1) Hasan Daneshmandi1, (1) Zahra AtrKarRoshan2 \\ 1 University of Guilan Faculty of Physical Education, Department of Sport Injury and Corrective Exercises, Guilan, Iran \\ 2University of Guilan Faculty of Medicine and Health Sciences, Department of Statistics, Guilan, Iran
}

\begin{abstract}
Objective: This study aimed to assist the realization of the postural control systems framework by presenting a short balance evaluation systems test (BESTest) that has a clear classification of items among postural control components to determine the cause of balance problems in older adults.

Materials and Methods: A total of 86 older adults with varying degrees of balance disorders performed all the BESTest items. An 11-member expert team participated in the content validity ratio study and item selection, and two evaluators determined the reliability of the scale made. Values of the area under the curve, sensitivity, and specificity were calculated.

Results: The short-BESTest consists of 12 selected items with a cut-off point of 20 out of 36 and a sensitivity of $72 \%$ and a specificity of $75 \%$. The test is moderately accurate at classifying participants with and without fall history. The values of interrater reliability (0.928) and concurrent validity $(r=0.926)$ were high $(p<0.01)$. Additionally, the short-BESTest approximately takes $12 \mathrm{~min}$.

Conclusion: Short-BESTest identifies the causes of balance deficits by classifying the postural control components. A low score in each of the short-BESTest sections indicates a defect in the postural control component of that section. Therefore, designing exercises using the short-BESTest to target the cause of the balance deficit can be performed better. Additionally, only section 5 of short-BESTest is used if a fall with low time and energy is to be predicted.
\end{abstract}

Keywords: Short balance evaluation systems test, posture, elderly, postural control

\section{Introduction}

Balance is an essential skill to avoid falls and balance disorders are common in older adults $(1,2)$. Because of the high incidence of balance disorders and their potential impact on performance, interventions to improve balance have become the primary goal of experts in this field (3-5). To this end, many functional balance assessment scales have been introduced (6-8).

Balance control is no longer considered just as a system or a set of balance and standing reflexes. Balance control is a complex skill consisting of the interaction of multiple sensorimotor processes (9). Based on this view, Horak (9) stated the key components needed for balance control in postural control systems framework. Horak (9) has described various components of postural control and emphasized the importance of each of these components in assessment and treatment.
In clinical practice and exercise design, all components needed for postural control should be studied and, according to disrupted components, exercise design and treatment should be performed \{Horak, 2006 \#508; Pourmahmoudian, 2020 \#543\}. One of the important limitations of all functional balance assessment scales introduced so far, except the BESTest (10), is that they do not include all postural control components and BESTest has so far been the only scale that includes all postural control components (6).

Another important limitation of functional balance assessment scales is that they are unable to determine the cause of the balance deficit (10). These scales provide a total score that, through the cut-off points, can generally determine whether a person is in danger of falling, and does not specify the type of postural control component that is impaired (10).

Address for Correspondence: Pedram Pourmahmoudian, University of Guilan Faculty of Physical Education, Department of Sport Injury and Corrective Exercises, Guilan, Iran

Phone: +989133731907 E-mail: ppourmahmoudian@gmail.com ORCID: orcid.org/0000-0001-6345-6759

Received: 14.12.2020 Accepted: 27.01.2021

Cite this article as: Pourmahmoudian P, Noraste AA, Daneshmandi H, AtrKarRoshan Z. Psychometric Properties of a Modified Balance Evaluation Systems Test. Eur J Geriatr Gerontol 2021;3(3):156-170

๑Copyright 2021 by the Academic Geriatrics Society / European Journal of Geriatrics and Gerontology published by Galenos Publishing House. 
BESTest has taken steps to remove this limitation and by dividing the items into six sections, it has attempted to determine the cause of the balance deficit to some extent (10). Although this segmentation is a very valuable step in determining the cause of the balance deficit, it has some drawbacks and cannot determine the cause of the balance deficit. In fact, BESTest lacks a clear and accurate segmentation of all postural control components to identify the causes of balance deficit, and the reasons why each item is included in one of these 6 BESTest sections are not stated. For example, in order to perform timed get up and go test with dual task, components of movement strategies, sensory strategies, dynamic control, and perception processing from the postural control system framework are involved in doing this item (6), but this item is only in the dynamic control section of BESTest (10). That is, if a person is unable to perform timed get up and go test with dual task, according to the BESTest segmentation, it only affects the dynamic control section score and the balance deficit is related to the dynamic control subcomponents.

In performing the functional reach test, components in sections of biomechanical constraints, movement strategies, sensory strategies, and orientation in space are involved in this motion (6), but this item is only in the biomechanical constraints section of BESTest (10). That is, if a person is unable to perform a functional reach test, according to this segmentation, the biomechanical constraints score is only affected and the balance deficit is related to the biomechanical constraint subcomponents. However, this conclusion is wrong. Horak (9) did not provide any reasons for this classification of items among the postural control sections. Perception processing is also a key component of the postural control system framework (9), but does not contain any section in BESTest and is not mentioned in the scoring system (10). Items that perception have a role in doing those are placed in "Stability in Gait" section of BESTest, if the balance deficit is due to the perception, how is it determined in BESTest?

So far, two mini-BESTest and brief-BESTest scales have been created as brief versions of BESTest. But the mini-BESTest does not evaluate the subcomponent of functional stability limits and the brief-BESTest doesn't evaluate the subcomponents of verticality and perception processing from the postural control system framework (6), and none of these tests provide a solution to achieve the cause of the balance deficit and it is only generally stated if the person has an impaired balance or not. Therefore, the purpose of this study is to assist in the realization of the postural control systems framework by presenting a short and modified BESTest that has a clear classification of items among postural control components to determine the cause of the balance problems.

\section{Materials and Methods}

\section{Participants}

A total of 86 participants [one 28-person group at the item selection stage (23 males and 5 females) with a mean age of $72.53 \pm 9.32$ and body mass index (BMI) of $24.13 \pm 2.59$, and a 58-person group at the confirmation stage (46 males and 12 females) with a mean age of $70.82 \pm 9.39$ and BMI of $24.69 \pm 2.72$ ] who lived in the community and nursing homes were studied with different ranges of balance disorders. Inclusion criteria included: Being over 60 years old, being able to walk 6 meters with or without assistive devices (but without human assistance) and completing a research consent form. Participants were introduced to a definition of fall (any disturbance of balance during daily activities that causes a person's trunk, knee or hand to lean against the ground, wall, desk or other surface unintentionally) and based on this definition; they self-reported the number of their falls last year.

The study was approved by the Guilan University Research Ethics Committee and was carried out in Physical Education College of Guilan University (ID: IR.GUMS.1397.021). An 11-member expert team (Table 1), including experts of physiotherapy, and physical education-sport injury and corrective exercises who specialize in assessing and treating balance problems, participated in the preparation of the short and modified BESTest (Table 2).

\section{Short-BESTest Preparation Process}

1. The conceptual framework in this research for the preparation of short-BESTest is the framework of postural control systems proposed by Horak (9) and is considered as the reference paper in this research.

2. As Sibley et al. (6) have shown, BESTest is the only test in which all postural control systems components are involved in performing its items. Since there are several items for each of the subcomponents of the postural control systems components in BESTest, BESTest was evaluated.

3. Classifying items among postural control components: In the reference paper of the postural control systems framework, the postural control subcomponents are divided into 6 sections (9). In Table 3, we set out the sections based on the reference article sections of the postural control systems framework (9), and identified which of the postural control subcomponents were involved in the implementation of each of these items.

4. Which postural control subcomponents are involved in the implementation of each item: This step was done by 11 experts in this research using the definitions of each of these subcomponents in the postural control systems reference paper (9), as well as the research by Sibley et al. (6) who determined sixty-six balance assessment scales are consisted of which components of postural control. For example, 
the subcomponents of stability limits (static stability) and strength from the biomechanical constraints section and the subcomponents of the sensory strategies section are involved in performing the one-leg stand test (6). The degrees of freedom and strength subcomponents of the biomechanical constraints section, the anticipatory and voluntary subcomponents of the motion strategies section, and the subcomponents of the sensory strategies section, are involved in performing the ankle strength and range of motion item (6).

5. Performing all BESTest items on 28 participants: 28 participants with different range of balance disorders, who had not previously performed BESTest, performed all BESTest items and their scores were given to 11 research experts for item selection.

6. Selecting items and summarizing BESTest: Items selected by experts should include all postural control components and obtain appropriate validity and reliability values. That is, there must be at least one item for each of the subcomponents of the postural control system components and this subcomponent must be involved in the implementation of this item. For example, among the thirty-six BESTest items, only the verticality subcomponent plays a role in performing these five items: BESTest 6 [lateral lean (left, right), sitting verticality (left, right)], and BESTest 20 (incline, eyes close). Therefore, at least one of these items should be selected to include the short-BESTest. Also, each section of the scale made should obtain good validity and reliability values. If each shortBESTest section obtained low values in validity, reliability, area under the curve (AUC), sensitivity and specificity, the items would have to be changed.

7. Based on the criteria outlined above, the content validity ratio (CVR) form for thirty-six BESTest items was completed by the experts. According to the number of the experts that was eleven, the CVR of 0.63 or above it indicates that the item is actually accepted (or 9 out of 11 experts must be in agreement) (11). The combination of items that achieve a CVR of 0.63 or above it creates the short-BESTest. $C V R=\frac{n e-\frac{N}{2}}{\frac{N}{2}}$

Table 1. Descriptive information of the research team and subjects

\section{Descriptive information of the subjects}

\begin{tabular}{l|l|l} 
Characteristics & Total $(n=58)$ & Without a history of falls $(n=36)$
\end{tabular}

\begin{tabular}{|l|l|l|}
\hline Age & $68.62 \pm 8.05$ & $68.44 \pm 8.29$ \\
\hline BMI & $25.09 \pm 3.13$ & $25.29 \pm 3.28$ \\
\hline BESTest & $79.02 \pm 19.34$ & $86.89 \pm 8.94$ \\
\hline Short-BESTest & $22.03 \pm 7.61$ & $24.86 \pm 4.47$ \\
\hline *: There is a significant difference between the faller group and the no-faller group \\
\hline
\end{tabular}

\section{Descriptive information of the research team}

\section{Expert no.}

1

2

3

4

5

6

7

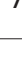

8

9

-

10

11
Scientific degree

Ph.D. in Physiotherapy - Professor of Department of Sport Injury and Corrective Exercises in University of Guilan, Iran

Ph.D. in Physical Education- Professor of Department of Sport Injury and Corrective Exercises in University of Guilan, Iran

Ph.D. in Physical Education- Professor of Department of Sport Injury and Corrective Exercises in University of Kerman, Iran

Ph.D. in Physiotherapy-Assistant Professor of Physiotherapy Department in University of Kermanshah, Iran

Ph.D. in Physiotherapy-Assistant Professor of Physiotherapy Department in University of Isfahan, Iran

Ph.D. in Physical Education - Assistant Professor of Department of Biomechanics, University of Guilan, Iran

Ph.D. in Physical Education - Assistant Professor of Department of Biomechanics, University of Hamadan, Iran

Ph.D. in Physical Education- Assistant Professor of Department of Sport Injury and Corrective Exercises in University of Yazd, Iran

Ph.D. student in Physical Education - Department of Sport Injury and Corrective Exercises in University of Guilan, Iran

Ph.D. student in Physical Education - Department of Sport Injury and Corrective Exercises in University of Guilan, Iran

Ph.D. student in Physical Education - Department of Sport Injury and Corrective Exercises in University of Guilan, Iran
Years of balance experience

22

18

11

10

11

2

1

4

2

1

3 
( $N=$ the number of panel members; ne=the number of experts agreeing "essential")

8. Defining the short-BESTest scoring system: Given that this scale is a short version of BESTest, no changes were made to the scoring system of each item. Items are scored on a sequential scale from 0 (the worst performance) to 3 (the best performance).
9. Performing BESTest on a 58-person group: After the items were selected based on the CVR form and scores of the first 28 people, another 58-person group performed the BESTest and $A U C$, sensitivity and specificity values were calculated for BESTest and short-BESTest using the scores of these participants (Table 4). Two experts independently were used to calculate the inter-rater reliability, and the reliability of each of the short-BESTest sections was also calculated (Table

\section{Table 2. Content validity ratio results of experts}

\begin{tabular}{|c|c|c|c|c|c|}
\hline No & Item & Essential & $\begin{array}{l}\text { Important, but } \\
\text { not essential }\end{array}$ & Not necessary & CVR \\
\hline 1 & BESTest 1 (Base of support) & 6 & 4 & 1 & 0.09 \\
\hline 3 & BESTest 3 (Ankle strength and ROM) & 10 & 1 & 0 & $0.81^{*}$ \\
\hline 4 & BESTest 4 (Hip/trunk lateral strength) & 8 & 1 & 2 & 0.45 \\
\hline 7 & BESTest 6 (Lateral lean-right) & 9 & 1 & 1 & $0.63^{*}$ \\
\hline 8 & BESTest 6 (Sitting verticality-left) & 6 & 1 & 4 & 0.09 \\
\hline 9 & BESTest 6 (Sitting verticality-right) & 6 & 1 & 4 & 0.09 \\
\hline 10 & BESTest 7 (Functional reach forward) & 10 & 1 & 0 & $0.81^{*}$ \\
\hline 14 & BESTest 10 (Rise to toes) & 7 & 2 & 2 & 0.27 \\
\hline 15 & BESTest 11 (Stand on one leg-left) & 11 & 0 & 0 & $1.00^{*}$ \\
\hline 16 & BESTest 11 (Stand on one leg-right) & 11 & 0 & 0 & $1.00^{*}$ \\
\hline 17 & BESTest 12 (Alternate stair touching) & 7 & 1 & 3 & 0.27 \\
\hline 18 & BESTest 13 (Standing arm raise) & 7 & 1 & 3 & 0.27 \\
\hline 19 & BESTest 14 (In-place response, forward) & 7 & 3 & 1 & 0.27 \\
\hline 20 & BESTest 15 (In-place response, backward) & 7 & 1 & 3 & 0.27 \\
\hline 21 & BESTest 16 (Compensatory stepping correction, forward) & 9 & 1 & 1 & $0.63^{*}$ \\
\hline 22 & BESTest 17 (Compensatory stepping correction, backward) & 7 & 2 & 2 & 0.27 \\
\hline 28 & BESTest 19-D (Stance on foam, eyes close) & 11 & 0 & 0 & $1.00^{*}$ \\
\hline 29 & BESTest 20 (Incline, eyes close) & 6 & 2 & 3 & 0.09 \\
\hline 30 & BESTest 21 (Gait, level surface) & 8 & 3 & 0 & 0.45 \\
\hline 31 & BESTest 22 (Change in gait speed) & 8 & 2 & 1 & 0.45 \\
\hline 32 & BESTest 23 (Walk with head turns, horizontal) & 10 & 1 & 0 & $0.81^{*}$ \\
\hline 33 & BESTest 24 (Walk with pivot turns) & 9 & 1 & 1 & $0.63^{*}$ \\
\hline 34 & BESTest 25 (Step over obstacles) & 9 & 1 & 1 & $0.63^{*}$ \\
\hline 35 & BESTest 26 (Timed "Get Up \& Go" test) & 8 & 2 & 1 & 0.45 \\
\hline 36 & BESTest 27 (Timed "Get Up \& Go" test with dual task) & 9 & 1 & 1 & $0.63^{*}$ \\
\hline
\end{tabular}


5). Short-BESTest concurrent validity was also calculated for BESTest (Table 6).

BESTest: It is composed of 36 items divided into 6 sections. Each item is scored from 0 (the most balance disturbance) to 3 (no balance disturbance) and the maximum possible score is 108 (10). BESTest measures all components of postural control systems and is the most comprehensive functional scale ever made to identify postural control disorders (6).

Postural control components: In this study, all the components of postural control are derived from the framework of postural control systems proposed by Horak (9), and each of these components has a comprehensive definition, and it is not correct to explain them briefly. Therefore, in this research, their explanation has not been discussed and we should refer to the reference article. It should be noted that the six sections of shortBESTest are exactly derived from the sections of the postural control framework (9), and are slightly different from the sections of BESTest. For example, in BESTest, the subcomponents of anticipatory postural adjustments and postural responses are placed as two separate sections (10), but in short-BESTest, these two subcomponents are in the section of movement strategies.

\section{Interrater Reliability and Concurrent Validity}

In this study, interrater reliability was obtained by two experts (one Ph.D. student in physical education -sport injury and corrective exercises with 2 years of balance experience and one master student in physical education- sport injury and corrective exercises with 1 year of balance experience), which was calculated with intra-class correlation coefficient (ICC). To teach the participants how to correctly perform each item, one examiner reads the item and the other examiner performs the item to teach them, then the subject performs the item. Each item was scored by two examiners independently. Participants were randomly assigned to each item to avoid the effect of fatigue on the last items. The participants were given short rest periods as needed.

ICC values were considered as follows: Between 0.9 to 0.99 as excellent reliability, between 0.75 to 0.9 as good reliability, between 0.75 to 0.5 as moderate reliability and less than 0.5 as poor reliability (12). Spearman correlation coefficient

\begin{tabular}{|c|c|c|c|c|c|c|}
\hline \multirow[b]{2}{*}{ Items } & \multicolumn{6}{|c|}{6 sections of short-BESTest } \\
\hline & $\begin{array}{l}\text { Section 1: } \\
\text { Biomechanical } \\
\text { constraints } \\
\text { (degrees of } \\
\text { freedom, strength, } \\
\text { limits of stability) }\end{array}$ & $\begin{array}{l}\text { Section 2: Movement } \\
\text { strategies (reactive, } \\
\text { anticipatory, } \\
\text { voluntary) }\end{array}$ & $\begin{array}{l}\text { Section 3: } \\
\text { Sensory strategies } \\
\text { (integration, } \\
\text { reweighting) }\end{array}$ & $\begin{array}{l}\text { Section 4: } \\
\text { Orientation } \\
\text { in space } \\
\text { (perception, } \\
\text { verticality) }\end{array}$ & $\begin{array}{l}\text { Section 5: } \\
\text { Control of } \\
\text { dynamics (gait, } \\
\text { proactive) }\end{array}$ & $\begin{array}{l}\text { Section 6: } \\
\text { Cognitive } \\
\text { processing } \\
\text { (attention, } \\
\text { learning) }\end{array}$ \\
\hline $\begin{array}{l}\text { Ankle strength and } \\
\text { ROM }\end{array}$ & $\begin{array}{l}\text { Degrees of freedom, } \\
\text { strength }\end{array}$ & Anticipatory, voluntary & $\begin{array}{l}\text { Integration, } \\
\text { reweighting }\end{array}$ & - & - & - \\
\hline $\begin{array}{l}\text { Lateral lean } \\
\text { (left, right) }\end{array}$ & Limits of stability & Anticipatory, voluntary & $\begin{array}{l}\text { Integration, } \\
\text { reweighting }\end{array}$ & $\begin{array}{l}\text { Orientation, } \\
\text { verticality }\end{array}$ & - & - \\
\hline $\begin{array}{l}\text { Functional reach } \\
\text { forward }\end{array}$ & $\begin{array}{l}\text { Limits of stability, } \\
\text { strength }\end{array}$ & Anticipatory, voluntary & $\begin{array}{l}\text { Integration, } \\
\text { reweighting }\end{array}$ & Orientation & - & - \\
\hline $\begin{array}{l}\text { Stand on one leg } \\
\text { (left, right) }\end{array}$ & $\begin{array}{l}\text { Limits of stability, } \\
\text { strength }\end{array}$ & - & $\begin{array}{l}\text { Integration, } \\
\text { reweighting }\end{array}$ & - & - & - \\
\hline $\begin{array}{l}\text { Compensatory } \\
\text { stepping correction, } \\
\text { forward }\end{array}$ & - & $\begin{array}{l}\text { Anticipatory } \\
\text { reactive }\end{array}$ & $\begin{array}{l}\text { Integration, } \\
\text { reweighting }\end{array}$ & Orientation & Proactive & - \\
\hline $\begin{array}{l}\text { Stance on foam, eyes } \\
\text { close }\end{array}$ & Limits of stability & - & $\begin{array}{l}\text { Integration, } \\
\text { reweighting }\end{array}$ & Orientation & - & - \\
\hline $\begin{array}{l}\text { Walk with head } \\
\text { turns, horizontal }\end{array}$ & - & Anticipatory, voluntary & $\begin{array}{l}\text { Integration, } \\
\text { reweighting }\end{array}$ & Orientation & Gait & Attention \\
\hline Walk with pivot turns & - & Anticipatory, voluntary & $\begin{array}{l}\text { Integration, } \\
\text { reweighting }\end{array}$ & Orientation & Gait & - \\
\hline Step over obstacles & $\begin{array}{l}\text { Degrees of freedom, } \\
\text { strength }\end{array}$ & Anticipatory, voluntary & $\begin{array}{l}\text { Integration, } \\
\text { reweighting }\end{array}$ & - & Gait & - \\
\hline $\begin{array}{l}\text { Timed "Get Up \& Go" } \\
\text { with dual task }\end{array}$ & - & Anticipatory, voluntary & $\begin{array}{l}\text { Integration, } \\
\text { reweighting }\end{array}$ & - & Gait & Attention \\
\hline \multicolumn{7}{|c|}{$\begin{array}{l}\text { Ability to orientate body parts regarding to the gravity, level of support, range of vision, and internal components are important parts of postural control (Horak, 2006), which in } \\
\text { "Orientation in space" section, orientation is used to express these abilities. } \\
\text { To distinguish each item in short-BESTest is consisted of what components of postural control systems, the } 11 \text { experts used the definitions of each of these components from the } \\
\text { postural control systems framework, (Horak, 2006) as well as the research by Sibley et al. (2015) who determined sixty-six balance assessment scales are consisted of which components } \\
\text { of postural control (Sibley et al. 2015) }\end{array}$} \\
\hline
\end{tabular}


was used to examine the validity of short-BESTest and its components with BESTest. Results 0 to 0.25 were considered as no correlation or very poor, 0.25 to 0.5 as poor correlation, 0.5 to 0.75 as moderate to good correlation, and above 0.75 as strong correlation (13).

\section{Statistics}

The subject's retrospective fall assessment was used as the gold standard in the diagnosis of a faller (i.e. one or more falls in the previous year) from non-faller (i.e. without any fall in the previous year). The receiver operating characteristic curve (ROC) was created for each balance scale (BESTest and short-BESTest) and the AUC of each ROC curve was calculated. The AUC is the probability of correctly identifying the faller from a pair of randomly selected patients (one faller and the other non-faller). The AUC range is 0.5 (non-detectable) to 1.00 (completely detectable). An AUC value of 0.9 and greater indicates high accuracy, 0.7 to 0.9 indicates moderate accuracy, 0.5 to 0.7 indicates low accuracy (14).
Sensitivity (number of correctly detected fallers) and specificity (number of correctly detected non-fallers) are calculated. Cutoff points were selected to distinguish between individuals with and without a history of falls using an intersection point that has the highest values of sensitivity and specificity (14).

Positive likelihood ratios are calculated as $\frac{\text { sensitivity }}{1-\text { specificity }}$. A positive likelihood ratio indicates how much the probability of being a faller increases with the positive test results. Negative likelihood ratios are calculated as $\frac{1-\text { sensitivity }}{\text { specificity }}$. The negative likelihood ratio indicates how much the probability of being a faller decreases with negative test results (14).

Data were analyzed using SPSS version 20 (SPSS Inc. Chicago, IL) and Stata 14 software (STATA, LIC. Texas). Significance level in the present study was $95 \%$ and alpha level was less than or equal to 0.05 .

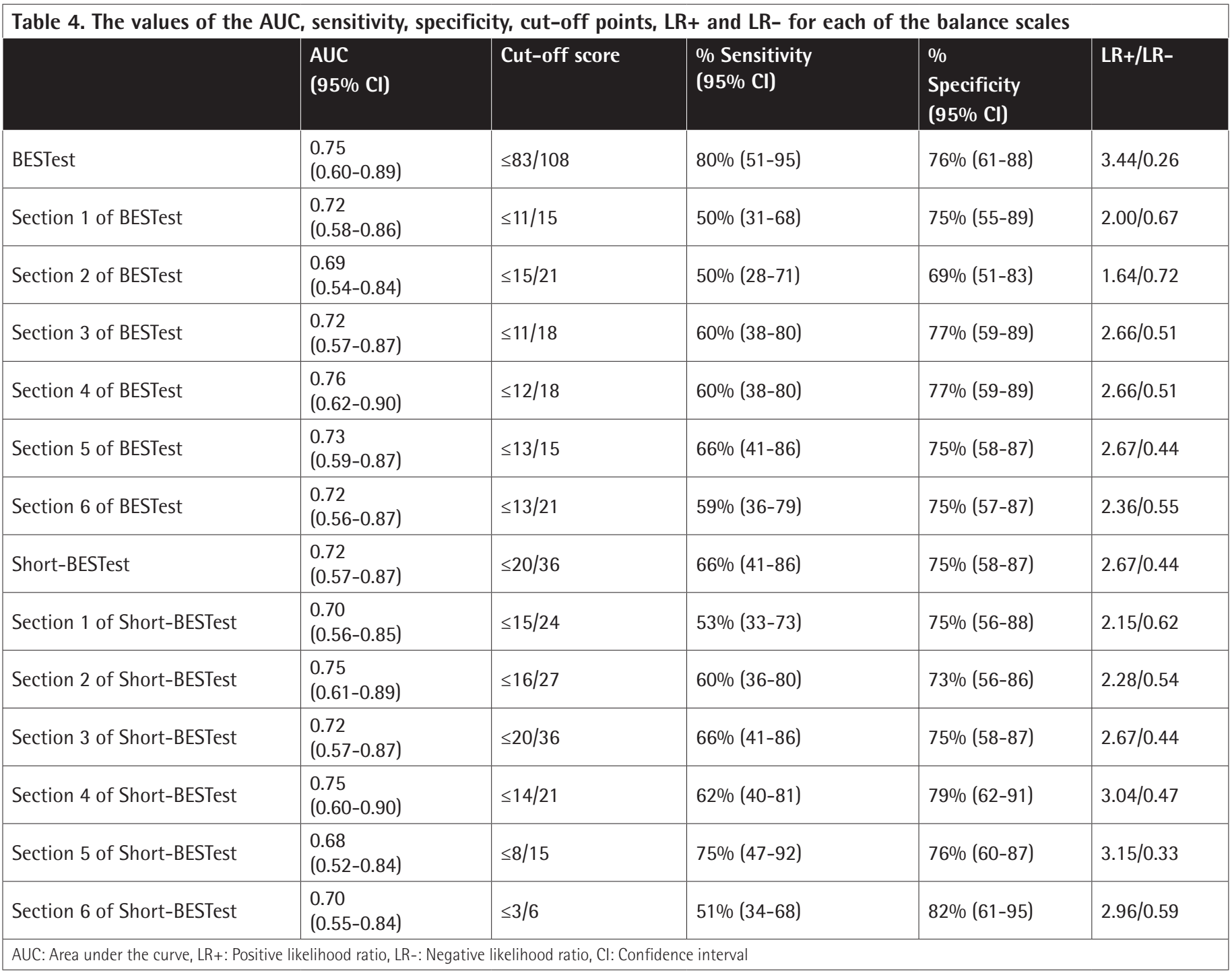




\section{Results}

Initially, 28 participants (23 males and 5 females) with a mean age of $72.53 \pm 9.32$ and BMI of $24.13 \pm 2.59$ performed all BESTest items and their scores were used to select short-BESTest items. Then, a group of 58 others (46 males and 12 females) who had not previously performed BESTest performed all of the BESTest items, and this group's scores are listed in this article (Table 1). In the group with a history of falling 6 participants had one fall and 16 participants had more than one fall in the past 12 months. Participants with and without history of falls exhibited significantly different scores for the BESTest $(p=0.001)$ and short-BESTest $(p=0.001)$.

Table 2 reports the results of expert surveys. As the number of experts is 11, the CVR score of 0.63 or above it means acceptance of the item, that 12 items totally were accepted.

Table 3 reports that each item in short-BESTest is consisted of what components of postural control systems. As can be seen in Table 3, all components of postural control systems are involved in short-BESTest. The components of sensory strategies are involved in the execution of all items, and the perception processing is involved in the execution of the 2 items.

Results from the ROC analyses are shown in Table 4. The BESTest, short-BESTest and all sections (except section 2 of BESTest and section 5 of short-BESTest) were moderately accurate at classifying participants with and without history of falls (between 0.70 and 0.76).

The cut-off points specified for BESTest are 83 out of 108 with $80 \%$ sensitivity and $76 \%$ specificity. Also, short-BESTest consists of 12 selected items with a cut-off point of 20 out of 36 and a sensitivity of $72 \%$ and a specificity of $75 \%$.

BESTest scores were more sensitive than the short-BESTest to identify older adults with balance disorders. The average of sensitivity between all sections of BESTest was 4\% lower than the average of short-BESTest sections, and the average of specificity between all sections of BESTest was $2 \%$ lower than the average of short-BESTest sections. In addition, the LR+ and LR- analyses of the BESTest, short-BESTest and all sections indicated that they are relatively similar for classifying participants with and without history of falls.

\section{Interrater Reliability}

The ICC results are presented in Table 5 to evaluate the interrater reliability of the short-BESTest. The inter-rater reliability of short-BESTest with ICC $=0.928$ was excellent $(p=0.001)$. Sections 1 and 3 also obtained excellent reliability values (0.911 and 0.909 ) and Sections 2, 4, 5 and 6 obtained good reliability values (0.834-0.893).

\begin{tabular}{|c|c|c|c|c|c|}
\hline & $\begin{array}{l}\text { First rater } \\
\text { (mean } \pm \text { SD) }\end{array}$ & $\begin{array}{l}\text { Second rater } \\
\text { (mean } \pm \text { SD) }\end{array}$ & $95 \% \mathrm{Cl}$ & ICC & $\mathbf{p}$ \\
\hline Section 1 of short-BESTest & $15.02 \pm 5.23$ & $14.67 \pm 4.05$ & $0.850-0.947$ & 0.911 & 0.001 \\
\hline Section 2 of short-BESTest & $18.21 \pm 5.43$ & $17.88 \pm 4.04$ & $0.819-0.937$ & 0.893 & 0.001 \\
\hline Section 5 of short-BESTest & $8.95 \pm 3.05$ & $9.10 \pm 2.40$ & $0.758-0.915$ & 0.856 & 0.001 \\
\hline Section 6 of short-BESTest & $3.28 \pm 1.24$ & $3.50 \pm 1.14$ & 0.720-0.902 & 0.834 & 0.001 \\
\hline
\end{tabular}

Table 6. Spearman correlation coefficient results to examine the relationship between short-BESTest and BESTest

\begin{tabular}{|l|l|l|l|}
\hline Short-BESTest with BESTest & $\begin{array}{l}\text { Spearman } \\
\text { correlation }\end{array}$ & $\mathbf{p}$ & $\begin{array}{l}\text { Number of } \\
\text { participants }\end{array}$ \\
\hline Section 1 of short-BESTest with BESTest & $0.926^{*}$ & 0.001 & 58 \\
\hline Section 2 of short-BESTest with BESTest & $0.864^{*}$ & 0.001 & 58 \\
\hline Section 3 of short-BESTest with BESTest & $0.912^{*}$ & 0.001 & 58 \\
\hline Section 4 of short-BESTest with BESTest & $0.941^{*}$ & 0.001 & 58 \\
\hline Section 5 of short-BESTest with BESTest & $0.900^{*}$ & 0.001 & 58 \\
\hline Section 6 of short-BESTest with BESTest & $0.855^{*}$ & 0.001 & 58 \\
\hline${ }^{*}$ : There was a significant relationship between the two variables $(p<0.001)$ & $0.819^{*}$ & 0.001 & 58 \\
\hline
\end{tabular}




\section{Concurrent Validity with BESTest}

To investigate the concurrent validity, the correlation between Short-BESTest and BESTest was calculated. Spearman correlation coefficient was 0.926 , which was significantly related with BESTest $(p<0.01)$ (Table 6$)$. All sections of shortBESTest also have a strong correlation with BESTest (0.8190.941).

\section{How to Enter the Scores Into the Short-BESTest Table? (Table 7)}

Each item is consisted of one or more postural control system components, so any scores obtained for each item are placed in front of those components, i.e. if the subject obtains 2 in doing the item of ankle strength and range of motion, score 2 is written in front of these sections: Biomechanical constraints, movement strategies, sensory strategies, and the short-BESTest total score. By summing the scores below each of the sections, the overall score for that section is obtained. Also by adding up the score for each item, the short-BESTest overall score is obtained.

\section{Discussion}

Using the values of the AUC, sensitivity, specificity, Spearman correlation coefficient and reliability the following 12 items were selected according to postural control system framework.

\section{Table 7. Short-BESTest scores calculation table}

\begin{tabular}{|c|c|c|c|c|c|c|c|c|}
\hline & \multirow[b]{2}{*}{ Items } & \multicolumn{6}{|c|}{6 Sections of short-BESTest } & \multirow[b]{2}{*}{$\begin{array}{l}\text { Short- } \\
\text { BESTest }\end{array}$} \\
\hline & & $\begin{array}{l}\text { Section 1: } \\
\text { Biomechanical } \\
\text { constraints } \\
\text { (degrees of } \\
\text { freedom, } \\
\text { strength, limits } \\
\text { of stability) }\end{array}$ & $\begin{array}{l}\text { Section 2: } \\
\text { Movement } \\
\text { strategies } \\
\text { (reactive, } \\
\text { anticipatory, } \\
\text { voluntary) }\end{array}$ & $\begin{array}{l}\text { Section 3: } \\
\text { Sensory } \\
\text { strategies } \\
\text { (integration, } \\
\text { reweighting) }\end{array}$ & $\begin{array}{l}\text { Section 4: } \\
\text { Orientation in } \\
\text { space (perception, } \\
\text { verticality) }\end{array}$ & $\begin{array}{l}\text { Section 5: } \\
\text { Control of } \\
\text { dynamics } \\
\text { (gait, } \\
\text { proactive) }\end{array}$ & $\begin{array}{l}\text { Section 6: } \\
\text { Cognitive } \\
\text { processing } \\
\text { (attention, } \\
\text { learning) }\end{array}$ & \\
\hline 1 & $\begin{array}{l}\text { Ankle strength } \\
\text { and ROM }\end{array}$ & & & & & & & \\
\hline 2 & $\begin{array}{l}\text { Lateral lean } \\
\text { (right) }\end{array}$ & & & & & & & \\
\hline 3 & $\begin{array}{l}\text { Lateral lean } \\
\text { (left) }\end{array}$ & & & & & & & \\
\hline 4 & $\begin{array}{l}\text { Functional reach } \\
\text { forward }\end{array}$ & & & & & & & \\
\hline 5 & $\begin{array}{l}\text { Stand on one } \\
\text { leg (right) }\end{array}$ & & & & & & & \\
\hline 6 & $\begin{array}{l}\text { Stand on one } \\
\text { leg (left) }\end{array}$ & & & & & & & \\
\hline 7 & $\begin{array}{l}\text { Compensatory } \\
\text { stepping } \\
\text { correction, } \\
\text { forward }\end{array}$ & & & & & & & \\
\hline 8 & $\begin{array}{l}\text { Stance on foam, } \\
\text { eyes close }\end{array}$ & & & & & & & \\
\hline 9 & $\begin{array}{l}\text { Walk with head } \\
\text { turns, horizontal }\end{array}$ & & & & & & & \\
\hline 10 & $\begin{array}{l}\text { Walk with pivot } \\
\text { turns }\end{array}$ & & & & & & & \\
\hline 11 & $\begin{array}{l}\text { Step over } \\
\text { obstacles }\end{array}$ & & & & & & & \\
\hline 12 & $\begin{array}{l}\text { Timed "Get Up } \\
\& \text { Go" with dual } \\
\text { task }\end{array}$ & & & & & & & \\
\hline \multicolumn{9}{|c|}{ Total of scores } \\
\hline \multicolumn{2}{|c|}{ Maximum scores } & 24 & 27 & 36 & 21 & 15 & 6 & 36 \\
\hline \multicolumn{2}{|c|}{ Cut-off score } & 15 & 16 & 20 & 14 & 8 & 3 & 20 \\
\hline
\end{tabular}


Ankle strength and ROM, lateral lean (right, left), functional reach forward, stand on one leg (right, left), compensatory stepping correction (forward), stance on foam (eyes close), walk with head turns (horizontal), walk with pivot turns, step over obstacles, timed "Get Up \& Go" with dual task.

In the results the sensitivity for BESTest and short-BESTest are $80 \%$ and $66 \%$, respectively, and the average of sensitivity between sections of BESTest is 4\% less than short-BESTest sections. In researches of Padgett et al. (15), Schlenstedt et al. (16), Duncan et al. (17), Godi et al. (18) and Yingyongyudha et al. (19) the values of sensitivity and specificity for BESTest, mini-BESTest and brief-BESTest have been reported by various researchers, that if we get the average value of them, we get $77.85,73.22$ and 67 for sensitivity, respectively, and $72.42,77.88$ and 83 for specificity. This indicates that as number of items decreases from BESTest, the sensitivity values decrease and the specificity values increase. Therefore, the decrease in the sensitivity of short-BESTest to BESTest is in line with the findings of other researchers (15-19). But the important point is that the sensitivity level is too low among the BESTest sections, especially sections 1 and 2 of this test (50\%, 50\%).

The low amounts of sensitivity in sections 1 and 6 of shortBESTest is other point. It shows components of biomechanical constraints (degrees of freedom, strength, limits of stability) and cognitive processing (attention, learning) have low sensitivity in elderly population, and section 5 of shortBESTest that is pertain to control of dynamics (gait, proactive) only with five items has good amounts of sensitivity (75\%) and specificity (76\%). It should also be noted that we must do all the items and sections of short-BESTest, because the reason for the problem of balance of an elderly person may be due to a defect in the subcomponents of section 1 or 6 of short-BESTest, and it should not be assumed that because in a whole society control of dynamic components can better predict fall occurrence, other components of postural control are not important. Rather it shows that the components of perception and biomechanical constraints are not good predictors of fall occurrence, but many older adults may have problems in these components. In general, if we want to predict a fall, we need to focus on dynamic control items, but to address the cause of the imbalance, each of these components must be considered.

The cut-off point specified for BESTest is similar to the research already specified (20). These cut-off points represent a superficial of the balance disorder associated with the risk of falling. Given the cut-off points selected, the short-BESTest was able to correctly identify 7 out of 10 fallers in this sample of the older adults, and it also correctly identified 7 out of 10 non-fallers. Also the high correlation between the BESTest and
short-BESTest supports the concurrent validity of the shortBESTest and its sections, and the inter-rater reliability of shortBESTest is approved.

One of the most important tasks for assessing balance status in the older adults is the construction and standardization of various tools including scales that can identify the causes of defects (10). Short-BESTest attempts to understand the causes of balance deficits by classifying the postural control components. One of key benefits of short-BESTest is that the researchers get familiar with the sub-components that have a role in doing each item, which enables the researchers to design exercises for all postural control components.

To eliminate the "being too long" constraint of BESTest, Franchignoni et al. (21) introduced its 14-item version (miniBESTest). By focusing on the dynamic balance, they eliminated two sections (biomechanical constraints and stability constraints) from the six BESTest sections. Although miniBESTest and BESTest have achieved relatively similar values of sensitivity, specificity and reliability in fall risk prediction (17), mini-BESTest is inconsistent with the postural control systems framework due to the removal of components from postural control systems (15). Padgett et al. (15) published the 8-item version (brief-BESTest) since mini-BESTest contradicts the BESTest theoretical framework. But they only focused on choosing the best item from each of the BESTest sections and did not notice that some of these sections were composed of several postural control components, so the components of vertical stability and perception processing are not considered in brief-BESTest (6).

Disruption of one or a combination of postural control subcomponents can lead to postural disorder. In designing the balance exercises, a dedicated exercise design should also be designed for each postural control components that is impaired $(9,10)$. So, using short-BESTest, the design of the exercises can be done more accurately. For example, if a person obtains a score less than 16 in "the movement strategies" section, this indicates that the subcomponents in this section are the cause of the balance deficit and special exercises should be performed to strengthen the subcomponents in this section. The subject may also score lower than the cut-off points in some sections of the scale and obtain appropriate points in several sections, so exercise designing should be done based on weak components.

Already many training protocols have been designed and implemented to improve balance, but most of them don't cover all postural control components (6). Most of these protocols aren't purposefully designed to remove the underlying cause of the balance deficit; this reduces the efficiency of the protocols $(9,10)$. Using short-BESTest, it is hoped that more targeted training protocols can be designed and improved. 


\section{Study Limitations}

In this study, we came up with a new classification to find the cause of balance deficit. However, one of the most important limitations of short-BESTest is that each section is composed of a number of sub-components, so this makes it difficult to identify the main cause of the balance defect. For example, the subcomponents of degrees of freedom, strength, and stability limits are in the biomechanical constraints section and the individual who is impaired in this section cannot determine which of these sub-components is most affected. So it might be better to increase the number of these sections and place each postural control source in a separate section.

Balance deficits need to be identified using short-BESTest and training protocols must be implemented for these balance deficits to determine the effectiveness of short-BESTest.

Because of religious limitations that there are between males and females in Iran, this study was mostly conducted on old men and the sample size of the women was small. Construct validity tests are need to determine whether the short-BESTest sections accurately identify discrete balance defects. It is still unclear to what extent sections 3 (sensory strategies) and 4 (orientation in space) make similar measurements and overlap. Further psychometric tests are needed to determine construct validity, concurrent validity, sensitivity, specificity, and the ability to guide the effective treatment for people with balance problems.

The classification of items among postural control sections is controversial. For example, in the single leg stance item, perception processing is also required, but this perception processing rate is low and as the difficulty of the test increases, the amount of perception processing increases (9). Therefore, only two items BESTest 23 (Walk with head turns, horizontal), and BESTest 27 (Timed "Get Up \& Go" with dual task) that require higher perception processing were considered perception processing items. This topic also applies to other items.

\section{Conclusion}

Short-BESTest is the second scale that includes all the components of the postural control systems and the most important advantage of the scale is that it determines each item is composed of which components of the postural control systems. So by using this classification, we can determine which component of the postural control systems is impaired and focus training programs on strengthening and improving this component. One of the other short-BESTest benefits is that it has fewer items than BESTest. Also it takes about 12 minutes to do the short-BESTest, compared to about 40 minutes to do the BESTest.

If we only want to predict the fall with low time and energy, we can only use section 5 of short-BESTest (control of dynamic), and in order to determine the cause of the balance defect, it is necessary to focus on all sections of short-BESTest and their cutting points, which indicates whether there is a defect in the subcomponents of each section or not.

\section{Acknowledgments}

We are very thankful to the subjects who made this study possible and who endured the inconvenience of this investigation.

\section{Ethics}

Ethics Committee Approval: The study was approved by the Guilan University Research Ethics Committee and was carried out in Physical Education College of Guilan University (ID: IR.GUMS.1397.021).

Informed Consent: Informed consent was obtained.

Peer-review: Externally peer-reviewed.

\section{Authorship Contributions}

Concept: P.P., A.A.N., H.D., Design: P.P., A.A.N., H.D., Data Collection or Processing: P.P., Z.A.K.R., Analysis or Interpretation: P.P., Z.A.K.R., Literature Search: P.P., A.A.N., Writing: P.P.

Conflict of Interest: All of authors have no conflicts of interest or financial or other contractual agreements that might cause conflicts of interest.

Financial Disclosure: The authors did not receive support from any organization for the submitted work.

\section{References}

1. Dillon CF, Gu Q, Hoffman HJ, Ko CW. Vision, hearing, balance, and sensory impairment in Americans aged 70 years and over: United States, 19992006. NCHS Data Brief 2010;1-8.

2. Tinetti ME, Kumar C. The patient who falls: "It's always a trade-off". JAMA 2010;303:258-266.

3. Gobbo S, Bergamin M, Sieverdes JC, Ermolao A, Zaccaria M. Effects of exercise on dual-task ability and balance in older adults: a systematic review. Arch Gerontol Geriatr 2014;58:177-187.

4. Hill KD, Hunter SW, Batchelor FA, Cavalheri V, Burton E. Individualized home-based exercise programs for older people to reduce falls and improve physical performance: A systematic review and meta-analysis. Maturitas 2015;82:72-84.

5. Rubenstein LZ. Falls in older people: epidemiology, risk factors and strategies for prevention. Age Ageing 2006;35(Suppl 2):ii37-ii41.

6. Sibley KM, Beauchamp MK, Van Ooteghem K, Straus SE, Jaglal SB. Using the systems framework for postural control to analyze the components of balance evaluated in standardized balance measures: a scoping review. Arch Phys Med Rehabil 2015;96:122-132.e29.

7. Berg K. Balance and its measure in the elderly: a review. Physiother Can 1989:41:240-246.

8. Pourmahmoudian $\mathrm{P}$, Noraste AA, Daneshmandi H, Atrkar Roshan Z. Functional balance assessment scales in elderly. Iranian Journal of Ageing 2018;13:132-153.

9. Horak FB. Postural orientation and equilibrium: what do we need to know about neural control of balance to prevent falls? Age Ageing 2006;35(Suppl 2):ii7-ii11. 
10. Horak FB, Wrisley DM, Frank J. The Balance Evaluation Systems Test (BESTest) to differentiate balance deficits. Phys Ther 2009;89:484-498.

11. Ayre C, Scally AJ. Critical values for Lawshe's content validity ratio: revisiting the original methods of calculation. Measurement and Evaluation in Counseling and Development 2014;47:79-86.

12. Koo TK, Li MY. A Guideline of Selecting and Reporting Intraclass Correlation Coefficients for Reliability Research. J Chiropr Med 2016;15:155-163.

13. Hubley AM, Zumbo BD. Validity and the consequences of test interpretation and use. Soc Indic Res 2011;103:219.

14. Akobeng AK. Understanding diagnostic tests 3: Receiver operating characteristic curves. Acta Paediatr 2007;96:644-647.

15. Padgett PK, Jacobs JV, Kasser SL. Is the BESTest at its best? A suggested brief version based on interrater reliability, validity, internal consistency, and theoretical construct. Phys Ther 2012;92:1197-1207.

16. Schlenstedt C, Brombacher S, Hartwigsen G, Weisser B, Möller B, Deuschl G. Comparing the Fullerton Advanced Balance Scale with the Mini-BESTest and Berg Balance Scale to assess postural control in patients with Parkinson disease. Arch Phys Med Rehabil 2015;96:218-225.

17. Duncan RP, Leddy AL, Cavanaugh JT, Dibble LE, Ellis TD, Ford MP, Foreman KB, Earhart GM. Comparative utility of the BESTest, mini-BESTest, and
brief-BESTest for predicting falls in individuals with Parkinson disease: a cohort study. Phys Ther 2013;93:542-950.

18. Godi M, Franchignoni F, Caligari M, Giordano A, Turcato AM, Nardone A. Comparison of reliability, validity, and responsiveness of the mini-BESTest and Berg Balance Scale in patients with balance disorders. Phys Ther 2013;93:158-167.

19. Yingyongyudha A, Saengsirisuwan V, Panichaporn W, Boonsinsukh R. The Mini-Balance Evaluation Systems Test (Mini-BESTest) Demonstrates Higher Accuracy in Identifying Older Adult Participants With History of Falls Than Do the BESTest, Berg Balance Scale, or Timed Up and Go Test. J Geriatr Phys Ther 2016;39:64-70.

20. Marques A, Almeida S, Carvalho J, Cruz J, Oliveira A, Jácome C. Reliability, Validity, and Ability to Identify Fall Status of the Balance Evaluation Systems Test, Mini-Balance Evaluation Systems Test, and Brief-Balance Evaluation Systems Test in Older People Living in the Community. Arch Phys Med Rehabil 2016;97:2166-2173.e1.

21. Franchignoni F, Horak F, Godi M, Nardone A, Giordano A. Using psychometric techniques to improve the Balance Evaluation Systems Test: the miniBESTest. J Rehabil Med 2010;42:323-331. 


\section{Short Balance Evaluation Systems Test}

\section{Examiner Instructions for Short-BESTest}

Subjects should be tested with flat heeled shoes or shoes and socks off. If subject must use an assistive device for an item, score that item one category lower. If subject requires physical assistance to perform an item score the lowest category (0) for that item.

\section{ANKLE STRENGTH \& RANGE}

(3) Normal: Able to stand on toes with maximal height and to stand on heels with front of feet up

(2) Impairment in either foot of either ankle flexors or extensors (i.e. less than maximum height)

(1) Impairment in two ankle groups (eg, bilateral flexors or both ankle flexors and extensors in 1 foot)

(0) Both flexors and extensors in both left and right ankles impaired (i.e. less than maximum height)

\section{LATERAL LEAN}

Left Right

(3) (3) Maximum lean, subject moves upper shoulders beyond body midline, very stable

(2) (2) Moderate lean, subject's upper shoulder approaches body midline or some instability

(1) (1) Very little lean, or significant instability

(0) (0) No lean or falls (exceeds limits)

\section{FUNCTIONAL REACH FORWARD}

Distance reached: $\mathrm{cm} \quad \mathrm{OR}$ inches

(3) Maximum to limits: $>32 \mathrm{~cm}$ (12.5 in)

(2) Moderate: $16.5 \mathrm{~cm}-32 \mathrm{~cm}(6.5-12.5 \mathrm{in})$

(1) Poor: $<16.5 \mathrm{~cm}(6.5 \mathrm{in})$

(0) No measurable lean - or must be caught

\section{STAND ON ONE LEG}

Left Right

(3) (3) Normal: Stable for $>20 \mathrm{~s}$

(2) (2) Trunk motion, OR 10-20 s

(1) (1) Stands $2-10 \mathrm{~s}$

(0) (0) Unable

\section{COMPENSATORY STEPPING CORRECTION- FORWARD}

(3) Recovers independently a single, large step (second realignment step is allowed)

(2) More than one step used to recover equilibrium, but recovers stability independently OR 1 step with imbalance

(1) Takes multiple steps to recover equilibrium, or needs minimum assistance to prevent a fall

(0) No step, OR would fall if not caught, OR falls spontaneously 


\section{STANCE ON FOAM SURFACE, EYES CLOSED}

(3) 30 s stable

(2) 30 s unstable

(1) $<30$ s

(0) Unable

\section{WALK WITH HEAD TURNS - HORIZONTAL}

(3) Normal: Performs head turns with no change in gait speed and good balance

(2) Mild: Performs head turns smoothly with reduction in gait speed

(1) Moderate: Performs head turns with imbalance

(0) Severe: Performs head turns with reduced speed AND imbalance AND/OR will not move head within available range while walking.

\section{WALK WITH PIVOT TURNS}

(3) Normal: Turns with feet close, FAST ( $<3$ steps) with good balance

(2) Mild: Turns with feet close SLOW (>4 steps) with good balance

(1) Moderate: Turns with feet close at any speed with mild signs of imbalance

(0) Severe: Cannot turn with feet close at any speed and significant imbalance

\section{STEP OVER OBSTACLES}

Time sec

(3) Normal: Able to step over 2 stacked shoe boxes without changing speed and with good balance

(2) Mild: Steps over 2 stacked shoe boxes but slows down, with good balance

(1) Moderate: Steps over shoe boxes with imbalance or touches box

(0) Severe: Cannot step over shoe boxes AND slows down with imbalance or cannot perform with assistance.

10. Timed "Get Up \& Go" With Dual Task Dual Task: Time $\sec$

(3) Normal: No noticeable change between sitting and standing in the rate or accuracy of backwards counting and no change in gait speed.

(2) Mild: Noticeable slowing, hesitation or errors in counting backwards OR slow walking (10\%) in dual task

(1) Moderate: Affects on BOTH the cognitive task AND slow walking (>10\%) in dual task

(0) Severe: Can't count backward while walking or stops walking while talking 


\section{INSTRUCTIONS FOR SHORT-BESTEST}

\section{ANKLE STRENGTH \& RANGE}

Examiner Instructions: Ask the patient rest their fingertips in your hands for support while they stand on their toes has high as possible and then stand on their heels. Watch for height of heel and toe lift.

Patient: Rest your fingers in my hands for support while you stand on your toes. Now stand on your heels by lifting up your toes. Maintain each position for $3 \mathrm{sec}$.

\section{VERTICALITY AND LATERAL LEAN}

Examiner Instructions: Patient is sitting comfortably on a firm, level, armless surface (bench or chair) with feet flat on floor. It is okay to lift ischium or feet when leaning. Watch to see if the patient returns to vertical smoothly without over or undershooting. Score the worst performance to each side Patient: Cross your arms over your chest. Place feet shoulder width apart. I'll be asking you to close your eyes and lean to one side as far as you can. You'll keep your spine straight, and lean sideways as far as you can without losing your balance OR using your hands. Keeping your eyes closed, return to your starting position when you've leaned as far as you can. It's okay to lift your buttocks and feet. Close your eyes Lean now (REPEAT other side).

\section{FUNCTIONAL REACH FORWARD}

Examiner Instructions: Examiner places the ruler at the end of the fingertips when the arms are out at 90 degrees. The patient may not lift heels, rotate trunk, or protract scapula excessively. Patient must keep their arms parallel to ruler and may use less involved arm. The recorded measure is the maximum horizontal distance reached by the patient. Record best reach

Patient: Stand normally. Please lift both arms straight in front of you, with fingertips held even. Stretch your fingers and reach forward as far as you can. Don't lift your heels. Don't touch the ruler or the wall. Once you've reached as far forward as you can, please return to a normal standing position. I will ask you to do this two times. Reach as far as you can

\section{STAND ON ONE LEG}

Examiner Instructions: Allow the patient two attempts and record the best. Record the sec they can hold posture, up to a maximum of $30 \mathrm{sec}$. Stop timing when subject moves their hand off hips or puts a foot down.

Patient: Look straight ahead. Keep your hands on your hips. Bend one leg behind you. Don't touch your raised leg on your other leg. Stay standing on one leg as long as you can. Look straight ahead. Lift now (REPEAT other side).

\section{COMPENSATORY STEPPING CORRECTION-FORWARD}

Examiner Instructions: Stand in front to the side of patient with one hand on each shoulder and ask them to push forward (Make sure there is room for them to step forward). Require them to lean until their shoulders and hips are in front of their toes. Suddenly release your support when the subject is in place. The test must elicit a step. Be prepared to catch patient.

Patient: Stand with your feet shoulder width apart, arms at your sides. Lean forward against my hands beyond your forward limits. When I let go, do whatever is necessary, including taking a step, to avoid a fall.

\section{SENSORY INTEGRATION FOR BALANCE (MODIFIED CTSIB)}

Examiner Instructions: Do the tests in order. Record the time the patient was able to stand in each condition to a maximum of 30 seconds. Repeat condition if not able to stand for $30 \mathrm{~s}$ and record both trials (average for category). Use medium density Temper ${ }^{\circledast}$ foam, 4 inches thick. Assist subject in stepping onto foam. Have the subject step off the foam between trials. Include leaning or hip strategy during a trial as "instability." 
Patient: For the next 4 assessments, you'll either be standing on this foam or on the normal ground, with your eyes open or closed. Place your hands on your hips. Place your feet together until almost touching. Look straight ahead. Each time, stay as stable as possible until I say stop.

\section{WALK WITH HEAD TURNS - HORIZONTAL}

Examiner Instructions: Ask the patient to turn their head and hold it so they are looking over their shoulder until you tell them to look over the opposite shoulder every 2-3 steps. If the patient has cervical restrictions allow combined head and trunk movements.

Patient: Begin walking at your normal speed, when I say "right", turn your head and look to the right. When I say "left" turn your head and look to the left. Try to keep yourself walking in a straight line.

\section{WALK WITH PIVOT TURNS}

Examiner Instructions: Demonstrate a pivot turn. Once the patient is walking at normal peed, say "turn and stop." Count the steps from turn until the subject is stable. Instability is indicated by wide stance width, extra stepping or trunk and arm motion.

Patient: Begin walking at your normal speed. When I tell you to "turn and stop", turn as quickly as you can to face the opposite direction and stop. After the turn, your feet should be close together.

\section{STEP OVER OBSTACLE}

Examiner Instructions: Place the 2 stacked boxes (9 inch or $22.9 \mathrm{~cm}$ height) $10 \mathrm{ft}$ away from where the patient will begin walking. Use a stopwatch to time gait duration to calculate average velocity by dividing the number of seconds into 20 feet. Look for hesitation, short steps and touch on obstacle.

Patient: Begin walking at your normal speed. When you come to the shoe boxes, step over them, not around them and keep walking.

\section{TIMED "GET UP \& GO" WITH DUAL TASK}

Examiner Instructions: Before beginning, practice with the patient how to count backward from a number between 90 and 100 by $3 \mathrm{~s}$, to make sure they can do the cognitive task. Then ask them to count backwards from a different number and after a few numbers say GO for the GET UP AND GO TASK. Time the patient from when you say "go" until they return to sitting. Stop timing when the patient's buttocks touch the chair bottom. The chair should be firm with arms to push from if necessary.

Patient: a) Count backwards by 3's starting at $100 \mathrm{OR}$ b) List random numbers and when I say "G0," stand up from the chair, walk at your normal speed across the tape on the floor, turn around, and come back to sit in the chair but continue listing numbers. 